\title{
Derivati dei nomi dei mesi (Febbraio)
}

\author{
Lucia Francalanci
}

PUBBLICATO: 28 FEBBRAIO 2021

\section{Quesito:}

Dopo quella sui derivati di gennaio, pubblichiamo la seconda scheda, relativa al mese di febbraio, della rubrica dedicata ai derivati dei nomi dei mesi. Per le informazioni generali si rimanda all'Introduzione.

\section{Derivati dei nomi dei mesi (Febbraio)}

I 1 nome febbraio deriva dal latino februarius, derivato di februus 'purificante', perché in tale mese si svolgevano riti di purificazione ed espiazione. Tra le varianti della forma toscana e poi italiana febbraio troviamo quelle antiche e regionali febbraro e febraro, che presentano l'esito non toscano -aro invece di -aio, e l'antico e letterario ferraio (con assimilazione). La forma febbraro è registrata da tutti i dizionari sincronici mentre febraro è accolta solo dal GDLI; il GDLI e il GRADIT registrano anche ferraio (il GDLI riporta anche il diminutivo ferraietto e il vezzeggiativo ferraiuzzo, entrambi con esempi tratti dai Proverbi toscani). L'Atlante Lessicale Toscano (ALT) mostra che la forma ferraio è attestata ancora negli anni '8o in tutta la regione, al di fuori della Lunigiana, dove prevale invece la variante ferraro.

Alla base dei derivati non sempre c’è la forma toscana e poi italiana febbraio, ma a volte anche febbraro.

- febbraieggiare/febbreggiare

Come gennareggiare, anche il verbo febbreggiare è proprio dei proverbi e si trova tipicamente nella forma se febbraio non febbreggia..., a indicare che il mese di febbraio non si comporta come dovrebbe, facendo il tempo caratteristico della stagione. Uno degli esempi riportati dai dizionari che registrano il termine (Tommaseo-Bellini, GDLI) è il proverbio toscano Se febbraio non febbreggia marzo campeggia [= 'se febbraio non è freddo, marzo è troppo rigoglioso, ci saranno troppe erbe'], presente anche nei dizionari di proverbi italiani (come Boggione e Massobrio 2004 o Lapucci 2006). Nell'Atlante Paremiologico Italiano sono attestate anche le varianti Se febbraio non febbrareggia... e Se febbraio non febbraresca. In Lapucci (2006) si trova anche la forma toscana febbraieggiare, in un proverbio che fornisce alcuni suggerimenti su come vestirsi durante i dodici mesi (in questo caso febbraieggia significa che febbraio un po' gela e un po' mostra il sole):

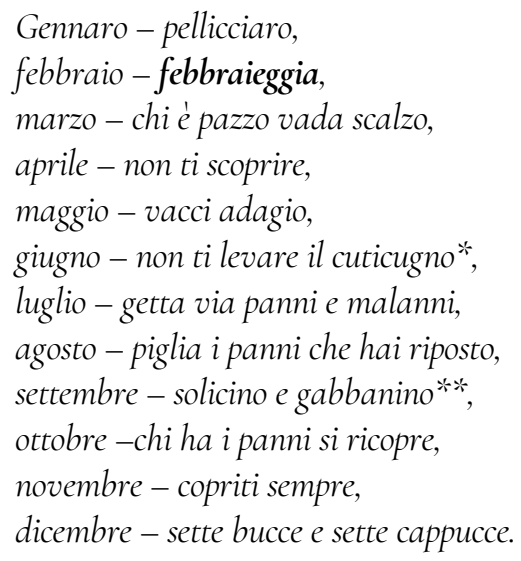


*'specie di giubbetto o sopravveste maschile $* *$ 'camice da lavoro utilizzato da contadini e operai'

\section{- febbraiese/febbrarese}

Come abbiamo visto per gennaiese/gennarese, è possibile rintracciare in rete alcuni aggettivi (anche sostantivati), non registrati dai dizionari sincronici, usati in riferimento ad alcune colture e i cui nomi derivano dal mese della raccolta. Cosi, ad esempio, il cavolfiore tipico della zona napoletana annovera tra le specie il febbraiese/febbrarese e la cipolla bianca comprende le tipologie febbrarese, aprilatica e giugnese:

Gli ecotipi locali differiscono principalmente per l'epoca di raccolta che dipende dal ciclo colturale e quindi dall'epoca di maturazione che va da febbraio a giugno. Gli ecotipi prendono infatti il nome dall'epoca di raccolta: Febbrarese, Marzatica, Aprilatica, Maggiaiola e Giugnese. In questo modo, il coltivatore può fornire un prodotto fresco per lungo tempo sul mercato, evitando problemi e costi dovuti alla conservazione. (Emanuela Fontana, Il valore nutraceutico delle cipolle Bianca di Pompei aumenta in primavera, FreshPlaza.it, I4/o3/2017)

\section{- febbraino/febbrarino}

Nel volume I giorni e le opere di Venezia (Treviso, Edizioni della Galleria, I985), il filologo Francesco Semi segnala la voce febbrarino, affermando che "questo aggettivo manca nei vocabolari, ma l'ho udito ripetere a Vicenza e Verona"; in realtà, l'aggettivo risulta attestato anche in italiano, almeno per quanto riguarda le occorrenze in rete. Se ne trova comunque traccia anche nella letteratura, come mostra l'esempio seguente:

C'era un po' di nebbia febbrarina, degli ultimi di febbraio, erano le primissime luci dell'alba ma la stazione era affollata come un veglione di carnevale, solo che non c'era musica, né danze, né stelle filanti. (Giorgio Scerbanenco, Il centodelitti, Garzanti, I97o, p. I82)

In rete è attestata anche la forma febbraino, associata soprattutto alle condizioni meteorologiche (aria febbraina, giorno febbraino, sera febbraina, sole febbraino, ecc.). Il termine è però principalmente usato come diminutivo di febbraio nella tradizione proverbiale: febbraino corto e malandrino (e varianti: febbraio febbraino, mese corto e malandrino; febbraio febbraino, freddo, corto e malandrino); febbraino ogni erba il suo capolino. Anche il diminutivo febbraietto si trova esclusivamente nelle sentenze proverbiali: febbraio febbraietto, mese corto e maledetto e variante febbraio febbraietto, corto e maledetto.

- febbraiolo/febbrainolo/febbrarolo

Febbraiuolo è registrato (soltanto) dal GDLI come aggettivo raro che significa 'di febbraio' o, in riferimento a un'erba o un fiore, 'che spunta in febbraio'. Nell'esempio fornito dal GDLI viene impiegata la variante febbraiolo:

Sopra un cuscino d'erba febbraiola / io vorrei coricarmi / soffiando tutti il mio fiato di vecchio / nella sottile calza della befana / lasciata dalla biscia tra le foglie. (C. Govoni, Preghiera al trifoglio, Roma, I95I, p.Io8)

L'aggettivo è presente anche nelle sentenze proverbiali nelle diverse varianti regionali. In Toscana ad esempio si hanno le forme febraiola e febbraiola: né d'erba febraiola né di donna festaiola non ti fidare e neve febbraiola dura una tosse e una cacaiola, ovvero 'rimane a lungo' (come ci spiega Lapucci 2006, p. Iori, "la misura del tempo allude al fatto che la tosse invernale è lunga e spesso sparisce solo con la 
stagione primaverile; il disturbo intestinale richiede per la guarigione un periodo di tempo non lungo, ma comunque con risentimenti continui"). Lo stesso proverbio si rintraccia in Veneto con la variante febrarola: la neve febrarola, la dura 'na cagarola.

Nel questionario dell'Atlante Paremiologico Italiano (2000) si trova anche la forma febbrarola:

Il primo la febbrarola

Il due la Candelora

Il tre san Biagio e le feste son passate;

risponde San mattia:

"ancora ci sono io

E a chi non guarda la mia festa

Gli spacco la mazza in testa".

L'aggettivo febbrai (u) olo è inoltre attestato con il significato di 'nato in febbraio', talvolta anche in forma sostantivata:

I nati nel mese di febbraio di Quart si sono ritrovati per trascorrere in allegria la serata dedicata ai "febbraioli". Con amici e parenti, una novantina di persone, i festeggiati hanno cenato e danzato all'hotel du Soleil di Ecloud per poi procedere all'elezione di "miss" e "mister" febbraioli ${ }_{9} 87$ (Quart, una miss per febbraio, "La Stampa", II/o2/1987).

\section{Cita come:}

Lucia Francalanci, Derivati dei nomi dei mesi (Febbraio), "Italiano digitale", 2021, XVI, 2021/1 (gennaio-marzo)

DOI: $10.35948 / 2532-9006 / 2021.5485$

\section{Copyright 2021 Accademia della Crusca}

Pubblicato con licenza creative commons CC BY-NC-ND 